Check for updates

Cite this: RSC Adv., 2017, 7, 29554

Received 21st April 2017

Accepted 1st June 2017

DOI: $10.1039 / c 7 r a 04504 b$

rsc.li/rsc-advances

\title{
Preparation and thermal properties of crosslinked polyurethane/lauric acid composites as novel form stable phase change materials with a low degree of supercooling
}

\begin{abstract}
Weibo Kong, (D) Xiaowei Fu, Ye Yuan, Zhimeng Liu and Jingxin Lei*
In this article, a series of crosslinked polyurethane/lauric acid composites was prepared as form stable phase change materials (FSPCMs) through a brief and solvent-free method. In the FSPCMs, lauric acid functioned as a phase change substance, and crosslinked polyurethane simultaneously functioned as a supporting material and phase change substance. Compared with traditional supporting materials in FSPCMs, the crosslinked polyurethane here obviously reduces the loss of latent heat from the supporting material and reduces the degree of supercooling of the obtained FSPCMs. The obtained FSPCMs were extensively studied by Fourier transform infrared spectroscopy (FTIR), scanning electron microscopy (SEM), X-ray diffraction (XRD), polarizing microscopy (POM), differential scanning calorimetry (DSC), thermal cycling tests and thermogravimetric analysis (TGA). The FTIR confirmed the chemical structure of the obtained FSPCMs. The XRD and POM results indicated that the FSPCMs exhibit comprehensive crystalline properties of polyurethane and lauric acid. DSC data showed that the FSPCMs have superior phase change properties with the phase change temperature and latent heat in the range of $26-38{ }^{\circ} \mathrm{C}$ and 90-131 $\mathrm{J} \mathrm{g}^{-1}$. Moreover, low or no supercooling of the obtained FSPCMs was detected by DSC. The phase change temperature is suitable in the fields of solar energy saving and building engineering. Thermal cycling tests and thermogravimetric analysis (TGA) proved the good thermal reliability and stability of the obtained FSPCMs.
\end{abstract}

\section{Introduction}

Thermal energy storage (TES) has attracted considerable attention due to the depletion of fossil fuels, climate issues and the energy crisis..$^{1-3}$ The employment of phase change materials (PCMs) is one promising way of storing thermal energy due to their high-energy density and small temperature fluctuation in the phase transition., ${ }^{4,5}$ Fatty acids and their mixtures have superior properties over the other investigated organic PCMs, such as high latent heat, low vapor pressure, no or less volume change during solid-liquid phase transition, low degree of supercooling, non-toxicity, and good thermal and chemical stability after long-term utilisation. ${ }^{6-8}$ Therefore, fatty acids have been extensively studied as promising PCMs for TES and have been applied in many areas such as solar energy applications, ${ }^{9}$ construction and building engineering, ${ }^{10}$ renewable energy saving applications ${ }^{11}$ and so forth.

It is well known that the fatty acid is a classical solid-liquid PCM, which must be encapsulated or sealed to prevent leakage

State Key Laboratory of Polymer Materials Engineering, Polymer Research Institute of Sichuan University, No. 24, First South Section First Ring Road, Wuhou District, Chengdu, 610065, China. E-mail: jxlei@scu.edu.cn during the phase change process. ${ }^{12,13}$ Cao and his group ${ }^{14}$ prepared a series of FSPCMs by absorbing liquid fatty acid eutectics into the carboxy methyl cellulose- 1 fibers. DSC results showed that the composites melt at $32.2{ }^{\circ} \mathrm{C}$ with latent heat of $114.6 \mathrm{~J} \mathrm{~g}^{-1}$ and solidify at $29.2^{\circ} \mathrm{C}$ with latent heat of $106.8 \mathrm{~J} \mathrm{~g}^{-1}$. Wei et al. ${ }^{15}$ developed expanded perlite/fatty acid composites as FSPCMs, and the latent heat values of composites are in the range of $82-89 \mathrm{~J} \mathrm{~g}^{-1}$. The supercooling degree of obtained FSPCMs exceeded $10^{\circ} \mathrm{C}$. Fu and his coworkers ${ }^{16}$ studied a kind of FSPCM based on lauric acid and diatomite, the melting and freezing temperature of the obtained FSPCM are $40.9{ }^{\circ} \mathrm{C}$ and $38.7^{\circ} \mathrm{C}$ with the latent heat of $57.2 \mathrm{~J} \mathrm{~g}^{-1}$. $\mathrm{He}^{17}$ reported fatty acid/ sludge ceramsite composite as FSPCM, and the melting temperature of the composite was $26.6^{\circ} \mathrm{C}$ with the corresponding latent heat of $47.1 \mathrm{~J} \mathrm{~g}^{-1}$. However, the fatty acids based FSPCMs with traditional materials such as expanded perlite, expanded graphite, diatomite and kaolin as supporting materials have significant disadvantage of low energy density and high degree of supercooling. ${ }^{18}$ The lower energy density of the PCM indicates the bigger necessary storage volume required in phase transition. Meanwhile, lower energy density demands a higher cost for the 

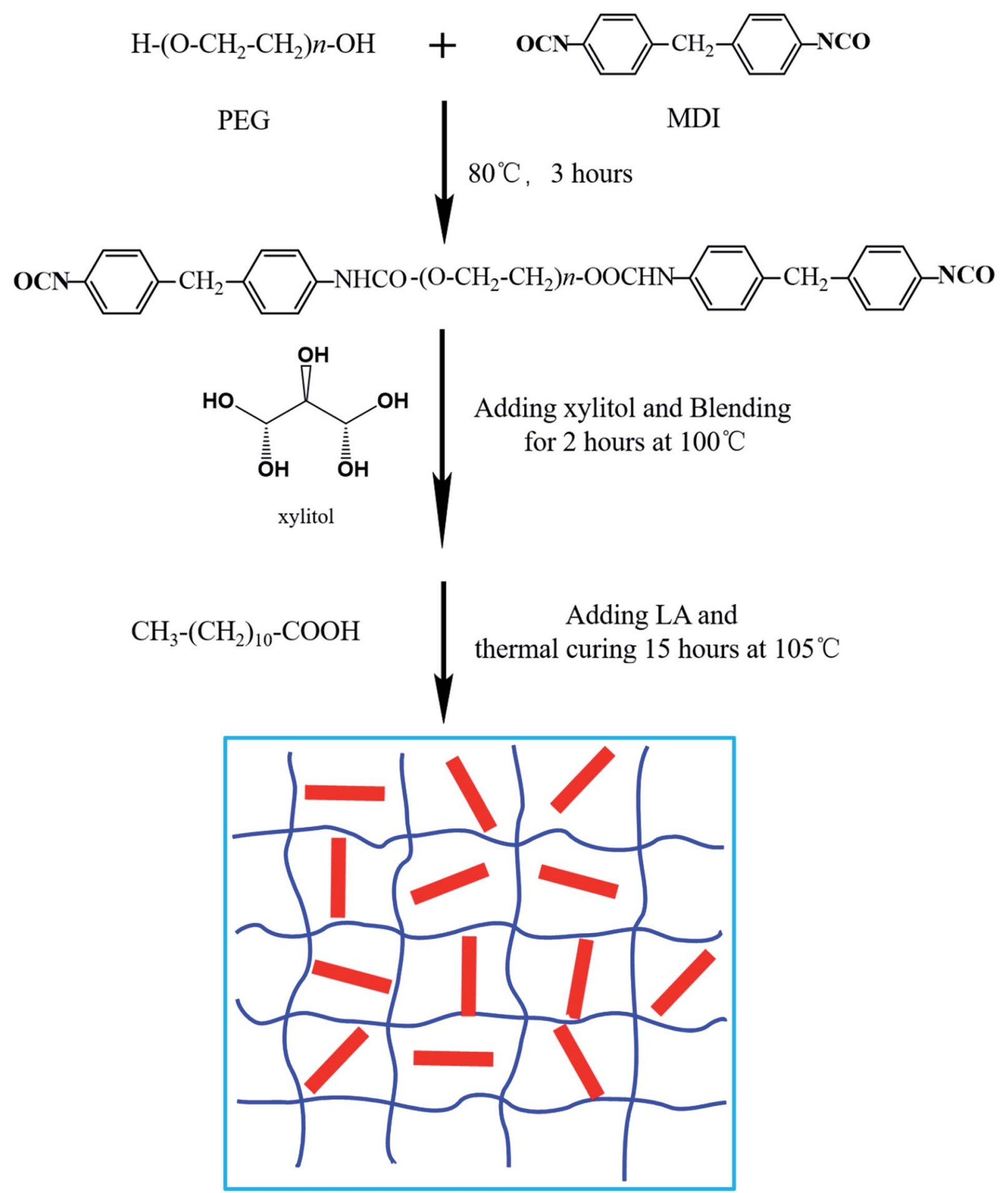

\section{LA}

\section{PU chains}

Fig. 1 The synthetic route of FSPCMs.

heat storage the same overall volume. ${ }^{19}$ High supercooling degree in PCM means large temperature difference between charging and discharging the latent heat, which is undesirable for efficient energy storage applications. ${ }^{\mathbf{2 0 - 2 3}}$ Therefore, it is necessary and significant to improve the energy density and reduce the degree of supercooling for PCMs. ${ }^{24}$ 
In this article, novel FSPCMs were prepared through solventfree and brief strategy with the lauric acid (LA) functioned as the phase change substance. The crosslinked PU based on the PEG4000 was selected as the supporting materials. Compared with the traditional supporting materials, the crosslinked PU

Table 1 The composition of PU and FSPCMs

\begin{tabular}{llllc}
\hline Sample & PEG4000 $(\mathrm{g})$ & MDI $(\mathrm{g})$ & Xylitol $(\mathrm{g})$ & LA $(\mathrm{g})$ \\
\hline PU & 40 & 5 & 0.608 & 0 \\
FSPCM-1 & 40 & 5 & 0.608 & 15 \\
FSPCM-2 & 40 & 5 & 0.608 & 30 \\
FSPCM-3 & 40 & 5 & 0.608 & 45 \\
FSPCM-4 & 40 & 5 & 0.608 & 60
\end{tabular}

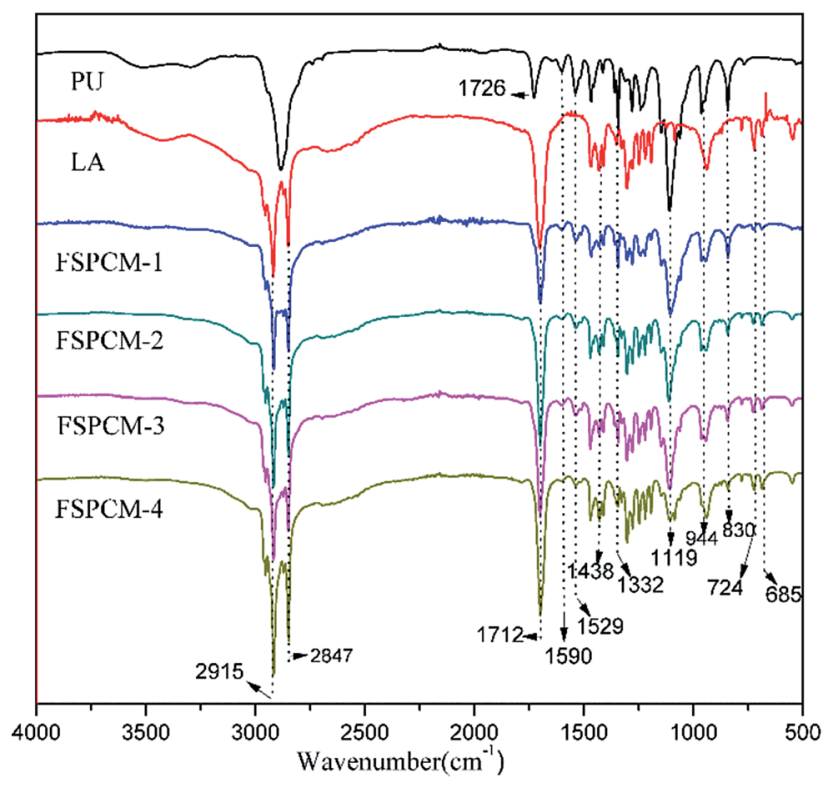

Fig. 2 The FTIR spectra of PU, LA and FSPCMs. employed in this article is also functioned as the phase change substance. Hence, PU here could significantly reduce the loss of latent heat from the supporting materials. Meanwhile, the degree of supercooling of obtained FSPCMs will decrease even vanish due to the overlap of the phase change process of PU and LA. The obtained FSPCMs were extensively studied by Fourier transform infrared spectroscopy (FTIR), scanning electron microscope (SEM), X-ray diffraction (XRD), polarizing microscope (POM), differential scanning calorimetry (DSC), and thermogravimetric analysis (TG), respectively.

\section{Experimental}

\section{Materials}

4,4'-Diphenylmethane diisocyanate (MDI) was provided by Yantai Wanhua polyurethane Co., Ltd. (Shandong, China). Polyethylene glycol (PEG, $M_{\mathrm{w}}=4000 \mathrm{~g} \mathrm{~mol}^{-1}$ ), xylitol and lauric acid (LA) were obtained from Kelong Chemical Reagent (Chengdu, China). The reagents above are used as received.

\section{Preparation of form stable PCMs (FSPCMs)}

0.01 mol PEG4000 and $0.02 \mathrm{~mol}$ MDI were putted into three neck flask equipped with thermometer and stirrer, and the polymerization continued for 3 hours under $80{ }^{\circ} \mathrm{C}$. Subsequently, $0.004 \mathrm{~mol}$ melted xylitol were added into prepolymer and blending for 2 hours at $100{ }^{\circ} \mathrm{C}$, after introduction of LA the mixture was transferred into an oven. Thermal curing reaction was performed at $105{ }^{\circ} \mathrm{C}$ for 15 hours before the FSPCMs were obtained. The detailed synthetic route is showed in Fig. 1.

PU, FSPCM-1, FSPCM-2, FSPCM-3 and FSPCM-4 represent the result products with different LA content, and the composition of PU and FSPCMs were listed in Table 1.

\section{Characterization}

The FTIR were conducted to study the chemical structure of LA, polyurethane (PU) and prepared FSPCMs. The FTIR measurements were performed on the Nicolet 560 (Nicolette Co., USA) in
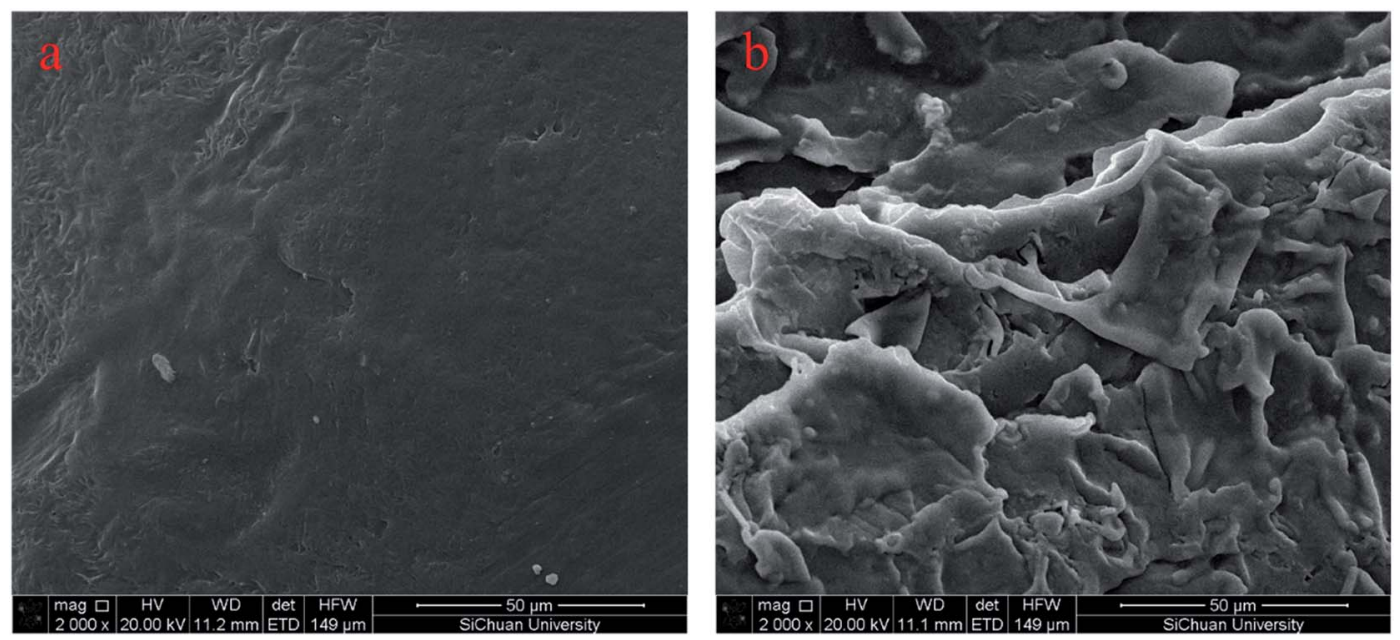

Fig. 3 The fracture surface morphology of (a) PU; (b) FSPCM-2. 


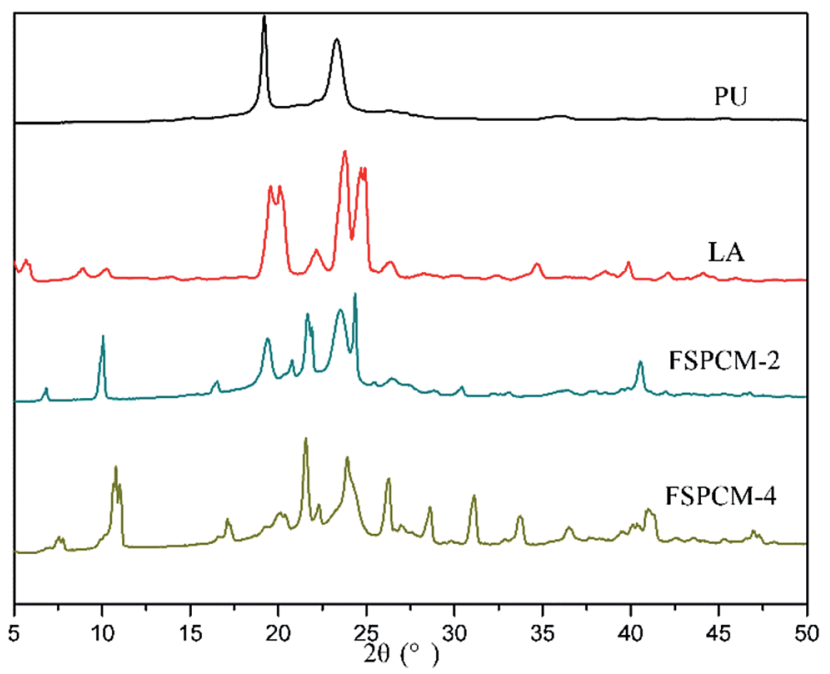

Fig. 4 The XRD spectra of PU, LA and FSPCMs. the scanning range of 4000 to $400 \mathrm{~cm}^{-1}$ with a resolution setting of $4 \mathrm{~cm}^{-1}$, and the prepared FSPCMs and PU were measured by attenuated total reflection model. The fracture surface morphology of pure PU and prepared FSPCM was detected on JEOL JSM-5900LV (Japan) at an accelerated voltage of $20 \mathrm{kV}$. Prior to the testing, the fracture surface of sample was puttercoated with gold powder. XRD measurements were conducted on Phillips X'Pert Pro MPD diffractometer in Bragg-Brentano geometry to study the crystalline properties of LA, PU and FSPCMs. The measurement data were collected in a range of $2 \theta$ $=5-50^{\circ}$ by a scanning rate of $0.04^{\circ} \mathrm{min}^{-1}$ at room temperature. Moreover, POM was employed to observe the crystalline morphology of LA and FSPCMs on the XPR-500D microscope (China) equipped with a video camera.

Phase change properties including phase change temperature, latent heat and supercooling degree of LA, PU and FSPCMs were recorded using the thermal analysis instrument DSC 204 (NETZSCH, Germany). The specimen was firstly heated from
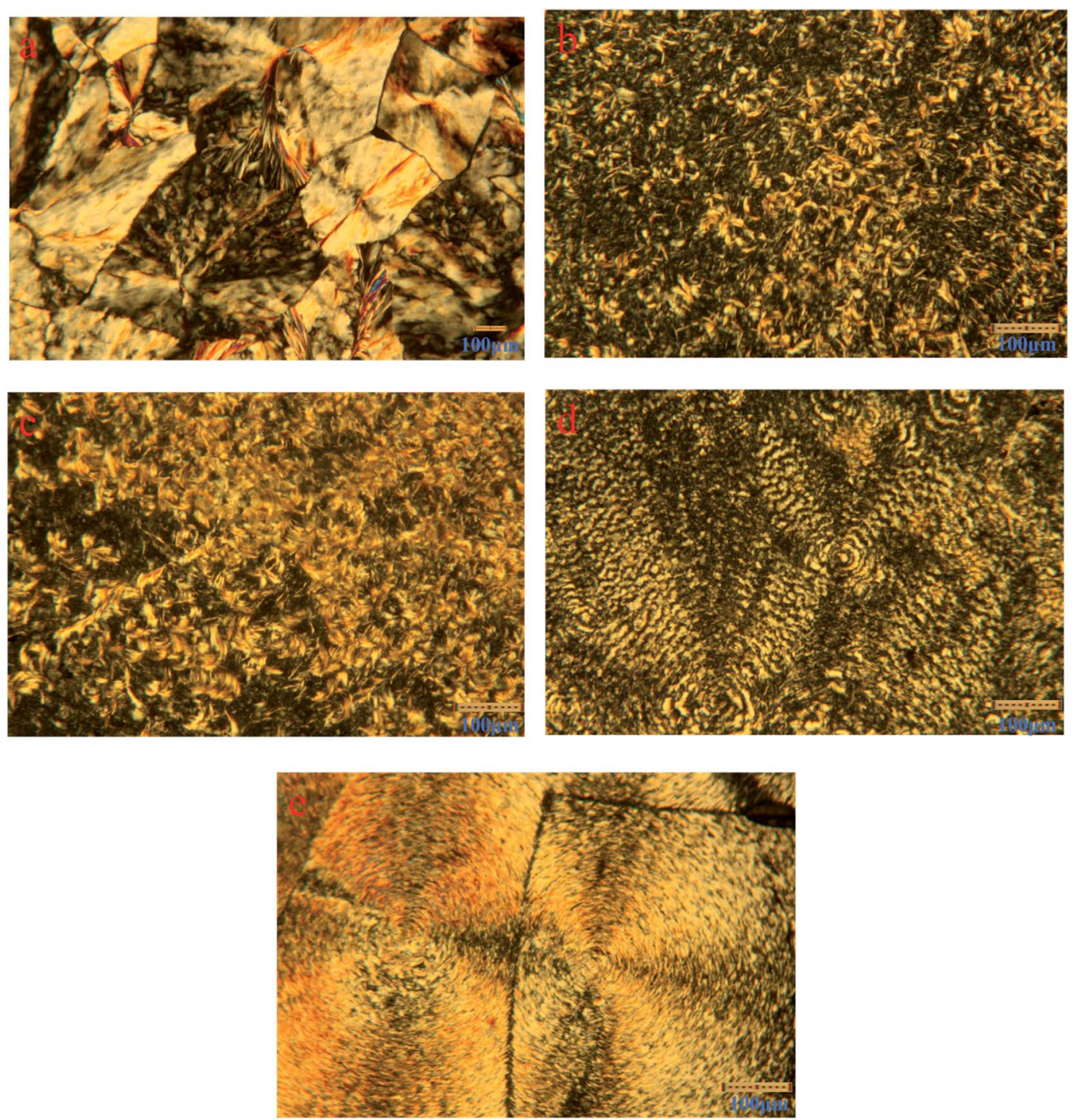

Fig. 5 The crystalline morphology of (a) LA; (b) FSPCM-1; (c) FSPCM-2; (d) FSPCM-3; (e) FSPCM-4. 
ambient temperature to $90{ }^{\circ} \mathrm{C}$ to erase prior heat history of the samples, then the specimen was cooled to $-20{ }^{\circ} \mathrm{C}$ to collect crystalline data. The specimen was lastly heated to $70{ }^{\circ} \mathrm{C}$ to record the melting data. Both heating and cooling temperature scans were carried out at the rate of $10{ }^{\circ} \mathrm{C} \mathrm{min}{ }^{-1}$ under a dry nitrogen atmosphere. Accelerated thermal cycling testing was conducted in a high-low temperature chamber to evaluate the thermal reliability of the prepared FSPCMs. The tests were performed with 100 consecutive heating/cooling processes with a heating rate of $3{ }^{\circ} \mathrm{C} \mathrm{min}^{-1}$ (in the temperature interval of 20
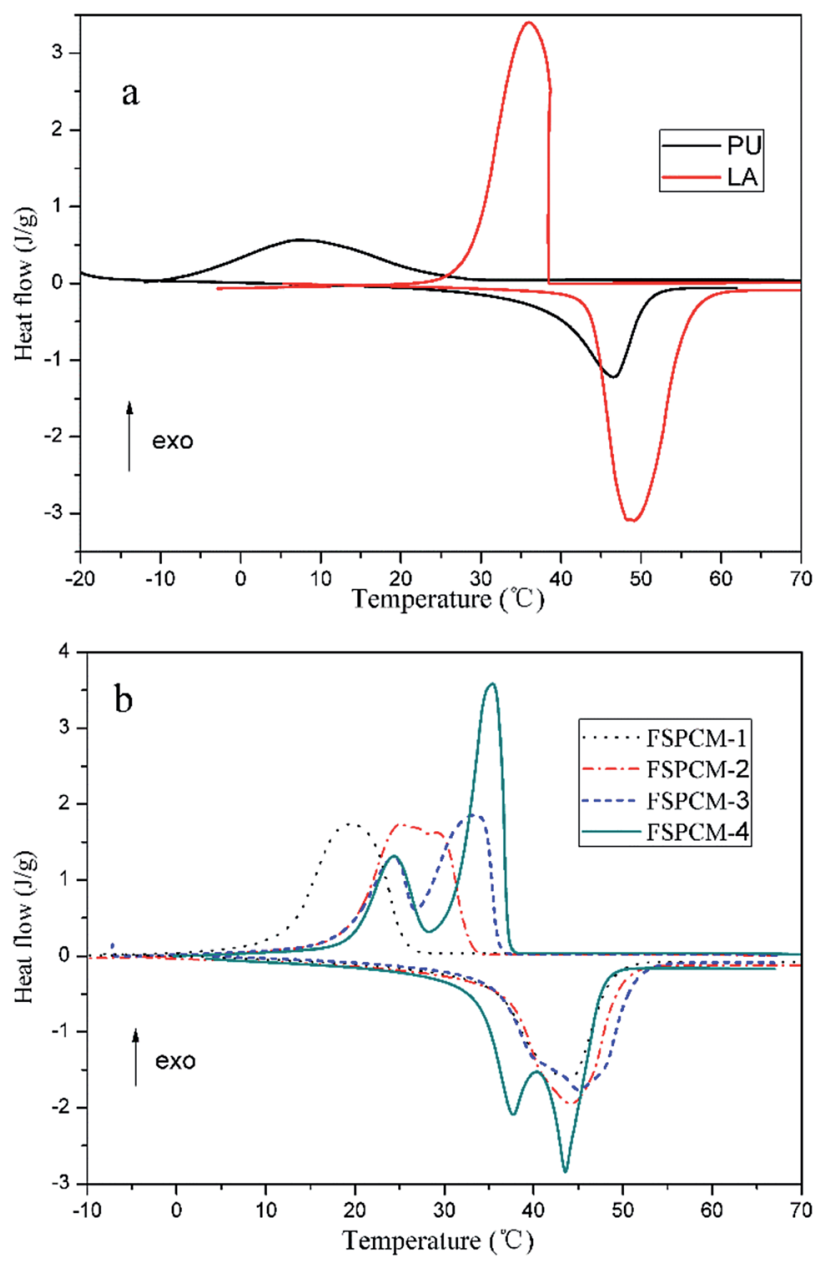

Fig. 6 The DSC results of PU, LA and FSPCMs.
$100{ }^{\circ} \mathrm{C}$ ). Subsequently, the phase change temperature and latent heat of thermal treated samples were assessed by DSC under previous testing condition. TGA measurements of LA, PU and FSPCMs were performed on thermogravimetry-differential thermal analysis instrument SDT-Q600 (TA, America) in the temperature range of $30-600{ }^{\circ} \mathrm{C}$ at a heating rate of $10{ }^{\circ} \mathrm{C} \mathrm{min} \mathrm{m}^{-1}$ under nitrogen atmosphere. The weight loss (TG) and digital thermogravimetry (DTG) were simultaneously recorded as a function of temperature.

\section{Results and discussion}

\section{Chemical structure}

FTIR are conducted to analyze the chemical structure and composition of FSCPCMs, and the FTIR spectra of PU, LA and FSPCMs are clearly shown in Fig. 2. As seen from Fig. 2, the FTIR spectrum of PU presents the characteristic stretching vibration peak of urethane group at $1726 \mathrm{~cm}^{-1}$ and $1529 \mathrm{~cm}^{-1}$, the distinct absorption peak of ether in PEG chains at $1119 \mathrm{~cm}^{-1}$. Moreover, the characteristic absorption peaks at $2878 \mathrm{~cm}^{-1}, 1332 \mathrm{~cm}^{-1}, 944$ $\mathrm{cm}^{-1}$ and $830 \mathrm{~cm}^{-1}$ are ascribed to $\mathrm{C}-\mathrm{H}$ vibration. For LA, characteristic peaks of $2915 \mathrm{~cm}^{-1}$ and $2847 \mathrm{~cm}^{-1}$ are attributed to $\mathrm{C}-\mathrm{H}$ stretching vibration, and the absorption peaks of carbonyl are recorded at $1712 \mathrm{~cm}^{-1}$. As shown in Fig. 2, distinct peaks at $2915 \mathrm{~cm}^{-1}, 2847 \mathrm{~cm}^{-1}, 1712 \mathrm{~cm}^{-1}$ and some other tiny peaks are detected in FSPCMs, results from the vibration of groups in LA, which indicates the existence of LA in prepared FSPCMs. The intensity of those peaks mentioned are positive relative to the LA content in FSPCMs. Meanwhile, the characteristic absorption peaks at $1590 \mathrm{~cm}^{-1}, 1529 \mathrm{~cm}^{-1}, 1119 \mathrm{~cm}^{-1}$ and $830 \mathrm{~cm}^{-1}$ belong to the vibration absorption of $\mathrm{PU}$ are also observed in prepared FSPCMs, implying the existence of PU in FSPCMs. The existence of the crosslinked PU plays a very important role in keeping solid state of FSPCM, when heated above LA's melting point, LA turns into molten state but can not translate freely. In addition, no new peaks occur in the spectra of FSPCMs, suggesting there is no chemical reaction between PU and LA.

\section{Morphology of fracture face}

SEM is performed to detect the micromorphology of the sample, and Fig. 3a and b demonstrate the fracture surface SEM images of PU and FSPCM-2, respectively. As shown in Fig. 3, the smooth and dark gray area are detected in SEM images of PU. Compared pure PU, FSPCM-2 exhibits obvious three-dimensional network

Table 2 Detailed DSC parameters of PU, LA and FSPCMs

\begin{tabular}{|c|c|c|c|c|c|c|c|}
\hline Sample & $\Delta H_{\mathrm{m}}\left(\mathrm{J} \mathrm{g}^{-1}\right)$ & $\Delta H_{\mathrm{m}-\mathrm{t}}\left(\mathrm{J} \mathrm{g}^{-1}\right)$ & $T_{\mathrm{m}}\left({ }^{\circ} \mathrm{C}\right)$ & $\Delta H_{\mathrm{c}}\left(\mathrm{J} \mathrm{g}^{-1}\right)$ & $\Delta H_{\mathrm{c}-\mathrm{t}}\left(\mathrm{J} \mathrm{g}^{-1}\right)$ & $T_{\mathrm{c}}\left({ }^{\circ} \mathrm{C}\right)$ & $\Delta T^{a}\left({ }^{\circ} \mathrm{C}\right)$ \\
\hline LA & 172.3 & - & 43.05 & 179.5 & - & 41.14 & 1.91 \\
\hline PU & 64.25 & - & 38.44 & 68.40 & - & 24.73 & 13.71 \\
\hline FSPCM-2 & 105.4 & 107.5 & 35.93 & 108.4 & 112.8 & 34.17 & 1.86 \\
\hline FSPCM-3 & 118.1 & 118.3 & 36.17 & 121.4 & 124.0 & 36.06 & 0.11 \\
\hline FSPCM-4 & 125.4 & 126.0 & 34.23 & 131.8 & 131.9 & 37.25 & -3.02 \\
\hline
\end{tabular}

${ }^{a} \Delta T=\mathrm{T}_{\mathrm{m}}-T_{\mathrm{c}}$. 
structure, and the dark gray area and brighter rugged structure on the surface are corresponding to PU and LA, respectively. The network structure of crosslinked PU will prevent the leakage of melted LA from FSPCM when the ambient temperature above the melting point of LA.

\section{Crystalline properties}

The XRD is conducted to study the crystalline properties of PU, LA and FSPCMs and corresponding diffraction spectra are presented in Fig. 4. Pure PU exhibits two strong and distinct peaks at $19.2^{\circ}$ and $23.3^{\circ}$, which is caused by the crystallization of PEG chains in PU. XRD diffraction peaks of LA are recorded at $8.78^{\circ}, 10.1^{\circ}, 19.6^{\circ}, 22.14^{\circ}, 23.84^{\circ}, 24.8^{\circ}$ and $26.2^{\circ}$. As for FSPCMs, the diffraction peaks of LA and pure PU are simultaneously detected, which means that the crystalline structure of FSPCMs is the combination of PU and LA. The XRD results indicates that the crystalline structure of LA and PU is not affected by the blending. However, the diffraction intensity of LA in FSPCMs is weaker than that of pure LA, which is attributed to the restricted movement of LA chains in FSPCMs.

POM is employed to further study the crystalline properties of FSPCMs, the POM images of LA and FSPCMs are presented in the Fig. 5. It can be seen from Fig. 5a, the LA shows obvious crystalline cross-extinction patterns (also reported in previous research ${ }^{25,26}$ ), which is different from that of PEG reported in previous study. ${ }^{27}$ Seen from Fig. $5 \mathrm{~b}$ and c, no obvious crystalline patterns are observed for FSPCM-1 and FSPCM-2, which must be ascribed to the restricted movement of LA chains in crosslinked structure of PU. The molecular movement ability of LA in FSPCMs will influence the degree of crystallization and the crystal size. Seen in Fig. $5 \mathrm{~d}$ and e, with the higher LA content in FSPCMs, the distinct cross-extinction patterns are observed in FSPCM-3 and FSPCM-4, and the crystal size exceed $150 \mu \mathrm{m}$, which suggests the good crystallization ability of LA in FSPCM-3 and FSPCM-4. This is because the restriction of crosslinked structure become weaker with the increasing of LA content, which can be further proved by the DSC results.

\section{Phase change properties}

Phase change properties determine the application value and serviceable range of PCM, so the phase change properties of $\mathrm{PU}$, LA and FSPCMs are extensively studied by DSC. The DSC curves of PU, LA and FSPCMs are given in the Fig. 6 and the detailed phase change temperature and latent heat are listed in Table 2. As shown in Fig. 6a, LA exhibits strong and sharp endothermic and exothermic peak with onset melting temperature $\left(T_{\mathrm{m}}\right)$ of $43.05{ }^{\circ} \mathrm{C}$ and onset crystallization temperature $\left(T_{\mathrm{c}}\right)$ of $41.14{ }^{\circ} \mathrm{C}$. The latent heat of melting $\left(\Delta H_{\mathrm{m}}\right)$ and crystallization $\left(\Delta H_{\mathrm{c}}\right)$ are 172.3 $\mathrm{J} \mathrm{g}^{-1}$ and $179.5 \mathrm{~J} \mathrm{~g}^{-1}$, indicating that LA is a good PCM with excellent phase change properties in the temperature range of $20-60{ }^{\circ} \mathrm{C}$. Conversely, PU has broad and blunt endothermic and exothermic peak with $T_{\mathrm{m}}$ of $38.44{ }^{\circ} \mathrm{C}$ and $T_{\mathrm{c}}$ of $24.73{ }^{\circ} \mathrm{C}$, and the corresponding latent heat are much less than that of LA. As seen from Fig. 6b, FSPCMs with different LA content exhibit endothermic and exothermic peak in the temperature range of $10-60{ }^{\circ} \mathrm{C}$, which indicate that the prepared FSPCMs have the reversible thermal energy storage and releasing ability in the phase change process. FSPCM-1 and FSPCM-2 exhibit single melting and freezing peak in each curve,
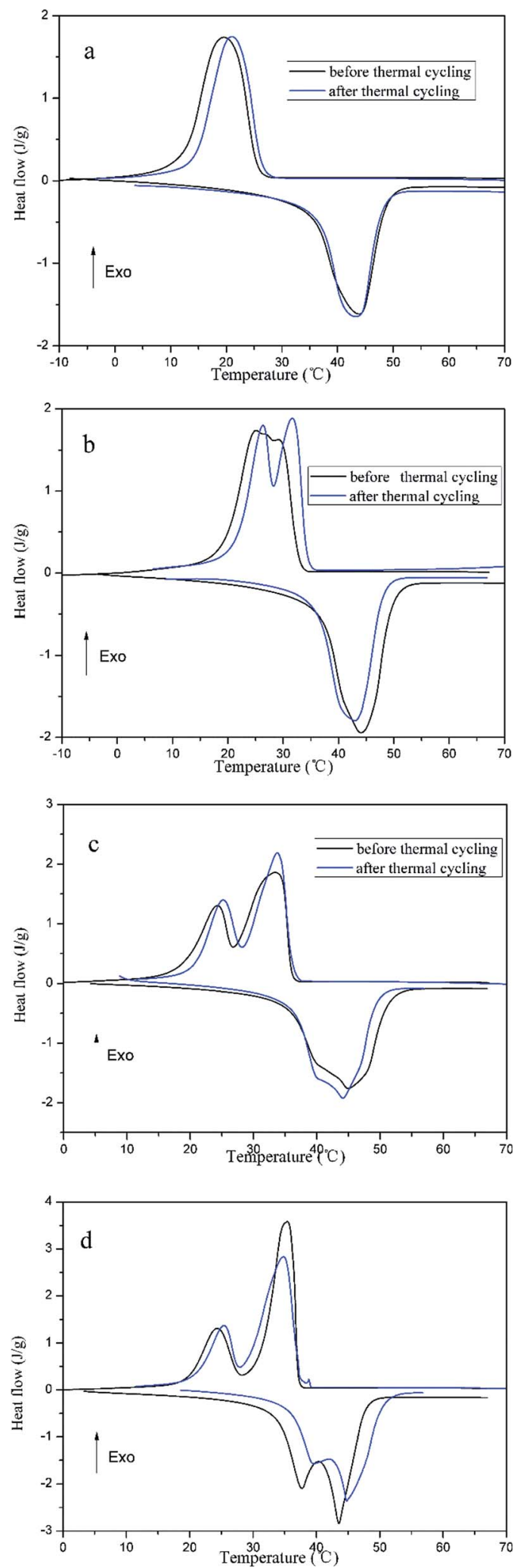

Fig. 7 The DSC curves of thermal treated FSPCMs, (a) FSPCM-1; (b) FSPCM-2; (c) FSPCM-3; (d) FSPCM-4. 
Table 3 Detailed DSC parameters of FSPCMs before and after 100 thermal cycling

\begin{tabular}{|c|c|c|c|c|c|c|c|c|}
\hline \multirow[b]{2}{*}{ Sample } & \multicolumn{2}{|c|}{$\Delta H_{\mathrm{m}}\left(\mathrm{J} \mathrm{g}^{-1}\right)$} & \multicolumn{2}{|l|}{$T_{\mathrm{m}}\left({ }^{\circ} \mathrm{C}\right)$} & \multicolumn{2}{|c|}{$\Delta H_{\mathrm{c}}\left(\mathrm{J} \mathrm{g}^{-1}\right)$} & \multicolumn{2}{|l|}{$T_{\mathrm{c}}\left({ }^{\circ} \mathrm{C}\right)$} \\
\hline & Before & After & Before & After & Before & After & Before & After \\
\hline FSPCM-2 & 105.4 & 103.0 & 35.93 & 36.69 & 108.4 & 105.5 & 34.17 & 32.64 \\
\hline FSPCM-3 & 118.1 & 113.3 & 36.17 & 35.38 & 121.4 & 117.5 & 36.06 & 35.91 \\
\hline FSPCM-4 & 125.4 & 123.1 & 34.23 & 39.96 & 131.8 & 130.7 & 37.25 & 37.03 \\
\hline
\end{tabular}

by contrast, the FSPCM-4 have two peaks in the cooling and heating curve. This may be due to the formation of two microphase in the FSPCM-4. Meanwhile, the endothermic and exothermic peak intensity of FSPCMs increase with the increasing of LA content, which is accordance with the latent heat value in Table 2. Seen from Table 2, each FSPCM has high latent heat exceeding $90 \mathrm{~J} \mathrm{~g}^{-1}$, which suggests that the prepared FSPCMs strong capability of absorbing or releasing latent heat during the phase change process. Especially for FSPCM-4, the melting and freezing latent heat are measured as $125.4 \mathrm{~J} \mathrm{~g}^{-1}$ and $131.8 \mathrm{~J} \mathrm{~g}^{-1}$ respectively, which indicate that the FSPCM with PU as supporting materials and LA as phase change substance exhibit excellent capacity of storing energy. Phase change temperatures ( $T_{\mathrm{m}}$ and $T_{\mathrm{c}}$ ) of FSPCMs are slightly lower than that of LA, this is possibly due to the higher degree of crystallinity and larger crystal size of LA compared with FSPCMs. This phenomenon is also caused by the restriction movement of LA in the FSPCMs, which is consistent with the results of XRD and POM.

In order to study the restricted movement of LA chains, the theoretical $\left(\Delta H_{\mathrm{t}}\right)$ and experimental $(\Delta H)$ latent heat value for FSPCMs are taken into comparison. $\Delta H_{\mathrm{m}-\mathrm{t}} / \Delta H_{\mathrm{c}-\mathrm{t}}$ is the theoretical latent heat of prepared FSPCMs for melting and crystallizing, which is calculated by the following formula:

$$
\begin{aligned}
\Delta H_{\mathrm{m}-\mathrm{t}}\left(\Delta H_{\mathrm{c}-\mathrm{t}}\right)= & \Delta H_{\mathrm{m}-\mathrm{PU}}\left(\Delta H_{\mathrm{c}-\mathrm{PU}}\right) \times \omega \mathrm{PU} \\
& +\Delta H_{\mathrm{m}-\mathrm{LA}}\left(\Delta H_{\mathrm{c}-\mathrm{LA}}\right) \times \omega \mathrm{LA}
\end{aligned}
$$

where $\Delta H_{\mathrm{m}-\mathrm{PU}}\left(\Delta H_{\mathrm{c}-\mathrm{PU}}\right)$ and $\Delta H_{\mathrm{m} \text {-PEG }}\left(\Delta H_{\mathrm{c}-\mathrm{PEG}}\right)$ are latent heat of PU and LA in the melting (crystallization) process respectively, $\omega \mathrm{PU}$ and $\omega \mathrm{LA}$ are $\mathrm{PU}$ and LA content in the FSPCMs respectively.

As can be seen from Table 2, the $\Delta H$ value is slightly lower than $\Delta H_{\mathrm{t}}$ value for FSPCMs, which further prove the adverse effect of crosslinked structure on the crystalline properties of LA. With the additional content of LA increase in FSPCMs, the difference between $\Delta H_{\mathrm{t}}$ and $\Delta H$ decrease. This is because the restriction of crosslinked structure on LA become weaker with the increasing of LA content, which is accordance with the POM results. Moreover, the $\Delta H$ value of FSPCM-3 and FSPCM-4 is almost close to $\Delta H_{\mathrm{t}}$ value, which show great practical value in the field of TES.

It is well known, supercooling is a thermophysical property of PCMs that is problematic in TES applications. ${ }^{28}$ The degree of supercooling $(\Delta T)$ is the temperature between the $T_{\mathrm{m}}$ and $T_{\mathrm{c}}{ }^{29}$ $\Delta T$ can be calculated by the equation: ${ }^{30}$

$$
\Delta T=T_{\mathrm{m}}-T_{\mathrm{c}}
$$

Seen in Fig. 6, the obvious supercooling phenomenon is detected in PU, LA, FSPCM-1 and FSPCM-2, however, there is no supercooling detected in FSPCM-3 and FSPCM-4, this is because the superposition of the LA and PU phase change process. The detailed $\Delta T$ are listed in Table 2, the $\Delta T$ of FSPCMs decreases even vanish from FSPCM-1 to FSPCM-4. Therefore, employing PU as supporting materials in obtained FSPCMs can put supercooling into operation, which will shorten temperature difference between charging and discharging the latent heat and improve the efficiency in energy storage applications.

The DSC results of prepared FSPCMs before and after thermal cycling are shown in Fig. 7, the corresponding phase
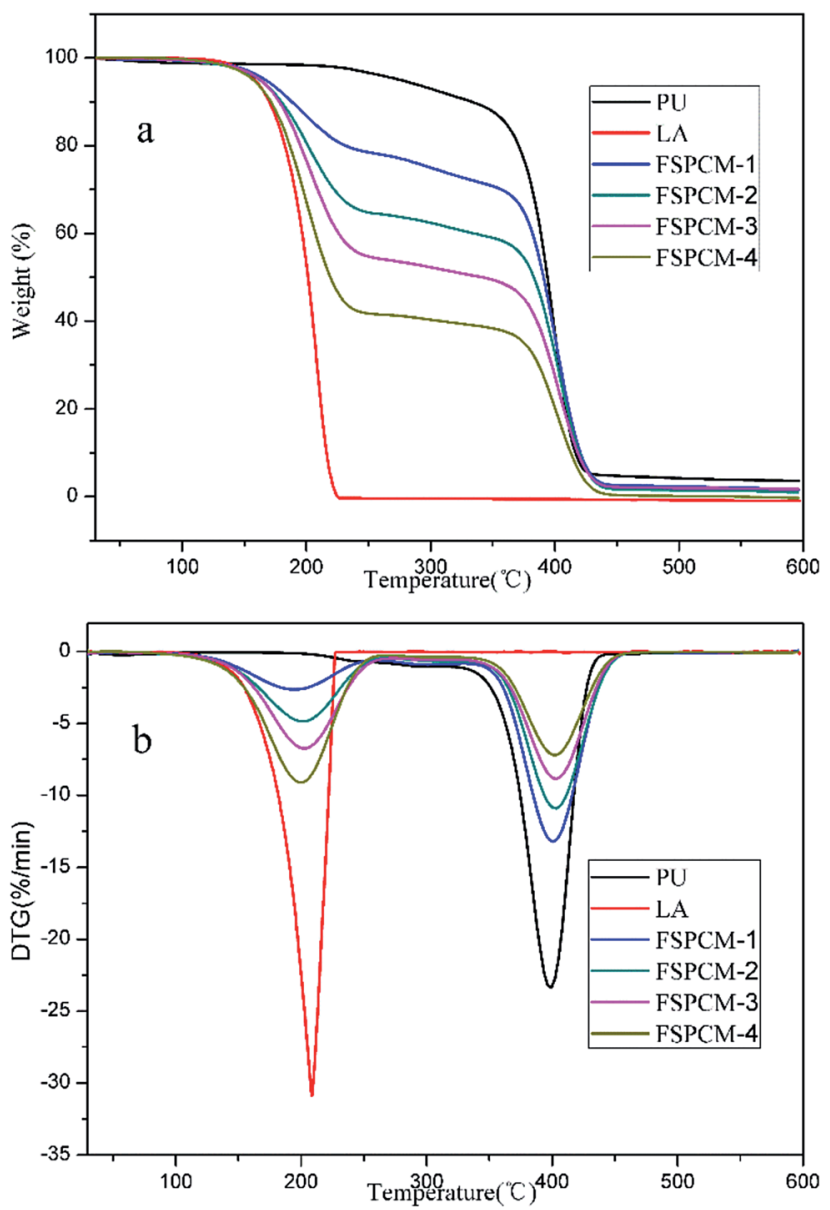

Fig. 8 The TG (a) and DTG (b) results of PU, LA and FSPCMs. 
change temperature and latent heat are listed in Table 3 . In Fig. 7, the DSC curves of FSPCMs before and after thermal treating are similar. As shown in Table 3, the $T_{\mathrm{m}}$ and $T_{\mathrm{c}}$ almost do not vary after thermal treating; the loss rate of latent heat value are limited in $5 \%$. The results indicate that the phase change temperature and latent heat are slightly affected by the thermal cycling, which means that the crosslinked structure of PU can prevent the leakage of LA during the thermal cycling. It can be draw a conclusion, the prepared FSPCMs have good thermal reliability with regard to the phase change temperature and latent heat.

\section{Thermal stability}

Thermal stability is one of the most important parameters for FSPCMs because the thermal decomposition temperature determines the application temperature range of FSPCMs. TG and DTG results of PU, LA and FSPCMs are shown in Fig. 8a and b, respectively. As shown in Fig. 8, LA and PU undergo one-step degradation process; FSPCMs exhibit two-step degradation mechanism. The first weight loss between $140{ }^{\circ} \mathrm{C}$ and $233{ }^{\circ} \mathrm{C}$ is attributed to the decomposition of LA, which is consistent with the degradation stage of pure LA. The second weight loss start at $358{ }^{\circ} \mathrm{C}$ is assigned to the degradation of PU. Meanwhile, the DTG peaks of FSPCM are detected at $208^{\circ} \mathrm{C}$ and $399^{\circ} \mathrm{C}$, which is similar to that of pure LA and PU. TGA results show that FSPCMs are thermal stable at the temperature below $140{ }^{\circ} \mathrm{C}$, which is higher than their working temperature (phase change temperature) and will ensure suitability for practical application. In addition, the TG results also present the LA content in FSPCMs, which is almost consistent with Table 1.

\section{Conclusion}

Novel FSPCMs based on PU and LA were prepared through a facile and solvent-free strategy. The LA functioned as the phase change substance, the PU simultaneously as supporting materials and phase change substance. The results showed that the crosslinked PU can prevent the leakage of LA during the phase change process. FTIR confirmed the chemical structure of obtained FSPCMs. POM and XRD results showed that the obtained FSPCMs exhibits crystalline properties of LA and PU. Meanwhile, the DSC results indicated that the obtained FSPCMs have superior phase change properties with high energy density in the temperature range of $26-38{ }^{\circ} \mathrm{C}$, and the supercooling degree of FSPCMs was decreased even eliminated due to the overlap of phase transition of LA and PU. TGA and thermal cycling test verified the thermal stability and reliability of obtained FSPCMs. It can be concluded that the obtained FSPCMs show great potential for TES.

\section{References}

1 R. Baetens, B. P. Jelle and A. Gustavsen, Phase change materials for building applications: A state-of-the-art review, Energ. Build., 2010, 42(9), 1361-1368.
2 K. Pielichowska and K. Pielichowski, Phase change materials for thermal energy storage, Prog. Mater. Sci., 2014, 65, 67123.

3 W. Kong, Y. Yang, C. Zhou and J. Lei, Novel thermosetting phase change materials with polycarbonatediol based curing agent as supporting skeleton for thermal energy storage, Energ. Build., 2017, 146, 12-18.

$4 \mathrm{~W}$. Kong, X. Fu, Z. Liu, C. Zhou and J. Lei, A facile synthesis of solid-solid phase change material for thermal energy storage, Appl. Therm. Eng., 2017, 117, 622-628.

5 Y. Zhang, L. Wang, B. Tang, R. Lu and S. Zhang, Form-stable phase change materials with high phase change enthalpy from the composite of paraffin and cross-linking phase change structure, Appl. Energy, 2016, 184, 241-246.

6 F. Tang, D. Su, Y. Tang and G. Fang, Synthesis and thermal properties of fatty acid eutectics and diatomite composites as shape-stabilized phase change materials with enhanced thermal conductivity, Sol. Energy Mater. Sol. Cells, 2015, 141, 218-224.

7 Ş. İnce, Y. Seki, M. Akif Ezan, A. Turgut and A. Erek, Thermal properties of myristic acid/graphite nanoplates composite phase change materials, Renewable Energy, 2015, 75, 243248.

8 S. Song, L. Dong, Y. Zhang, S. Chen, Q. Li, Y. Guo, et al., Lauric acid/intercalated kaolinite as form-stable phase change material for thermal energy storage, Energy, 2014, 76, 385-389.

9 J. Zeng, S. Zheng, S. Yu, F. Zhu, J. Gan, L. Zhu, et al., Preparation and thermal properties of palmitic acid/ polyaniline/exfoliated graphite nanoplatelets form-stable phase change materials, Appl. Energy, 2014, 115, 603-609.

10 A. Sarl, Fabrication and thermal characterization of kaolinbased composite phase change materials for latent heat storage in buildings, Energ. Build., 2015, 96, 193-200.

11 W. Liang, G. Zhang, H. Sun, P. Chen, Z. Zhu and A. Li, Graphene-nickel/n-carboxylic acids composites as formstable phase change materials for thermal energy storage, Sol. Energy Mater. Sol. Cells, 2015, 132, 425-430.

12 R. K. Sharma, P. Ganesan, V. V. Tyagi, H. S. C. Metselaar and S. C. Sandaran, Developments in organic solid-liquid phase change materials and their applications in thermal energy storage, Energy Convers. Manage., 2015, 95, 193-228.

13 H. Ke, Z. Pang, B. Peng, J. Wang, Y. Cai, F. Huang, et al., Thermal energy storage and retrieval properties of formstable phase change nanofibrous mats based on ternary fatty acid eutectics/polyacrylonitrile composite by magnetron sputtering of silver, J. Therm. Anal. Calorim., 2016, 123(2), 1293-1307.

14 L. Cao, Y. Tang and G. Fang, Preparation and properties of shape-stabilized phase change materials based on fatty acid eutectics and cellulose composites for thermal energy storage, Energy, 2015, 80, 98-103.

15 T. Wei, B. Zheng, J. Liu, Y. Gao and W. Guo, Structures and thermal properties of fatty acid/expanded perlite composites as form-stable phase change materials, Energ. Build., 2014, 68, 587-592. 
16 X. Fu, Z. Liu, Y. Xiao, J. Wang and J. Lei, Preparation and properties of lauric acid/diatomite composites as novel form-stable phase change materials for thermal energy storage, Energ. Build., 2015, 104, 244-249.

17 H. He, P. Zhao, Q. Yue, B. Gao, D. Yue and Q. Li, A novel polynary fatty acid/sludge ceramsite composite phase change materials and its applications in building energy conservation, Renewable Energy, 2015, 76, 45-52.

18 Y. Zhang, L. Wang, B. Tang, R. Lu and S. Zhang, Form-stable phase change materials with high phase change enthalpy from the composite of paraffin and cross-linking phase change structure, Appl. Energy, 2016, 184, 241-246.

19 A. F. Regin, S. C. Solanki and J. S. Saini, Heat transfer characteristics of thermal energy storage system using PCM capsules: A review, Renewable Sustainable Energy Rev., 2008, 12(9), 2438-2458.

$20 \mathrm{~J}$. Su and P. Liu, A novel solid-solid phase change heat storage material with polyurethane block copolymer structure, Energy Convers. Manage., 2006, 47(18-19), 31853191.

21 M. Faucheux, G. Muller, M. Havet and A. LeBail, Influence of surface roughness on the supercooling degree: Case of selected water/ethanol solutions frozen on aluminium surfaces, Int. J. Refrig., 2006, 29(7), 1218-1224.

22 X. J. Zhang, P. Wu, L. M. Qiu, X. B. Zhang and X. J. Tian, Analysis of the nucleation of nanofluids in the ice formation process, Energy Convers. Manage., 2010, 51(1), 130-134.

23 M. Dannemand, J. Dragsted, J. Fan, J. B. Johansen, W. Kong and S. Furbo, Experimental investigations on prototype heat storage units utilizing stable supercooling of sodium acetate trihydrate mixtures, Appl. Energy, 2016, 169, 72-80.

24 R. Al-Shannaq, J. Kurdi, S. Al-Muhtaseb, M. Dickinson and M. Farid, Supercooling elimination of phase change materials (PCMs) microcapsules, Energy, 2015, 87, 654-662.

25 J. Guo, H. X. Xiang, Q. Q. Wang and D. Z. Xu, Preparation and Properties of Polyacrylonitrile Fiber/Binary of Fatty Acids Composites as Phase Change Materials, Energy Sources, Part A, 2013, 35(11), 1064-1072.

26 J. Guo, X. K. You, L. Zhang, H. X. Xiang, S. Zhang and Y. F. Liu, Properties of a Solid-Solid Phase Change Material PAN/SA-LA/ZMS, Appl. Mech. Mater., 2014, 703, 3-8.

27 X. Fu, Y. Xiao, K. Hu, J. Wang, J. Lei and C. Zhou, Thermosetting solid-solid phase change materials composed of poly(ethylene glycol)-based two components: Flexible application for thermal energy storage, Chem. Eng. J., 2016, 291, 138-148.

28 A. Safari, R. Saidur, F. A. Sulaiman, Y. Xu and J. Dong, A review on supercooling of Phase Change Materials in thermal energy storage systems, Renewable Sustainable Energy Rev., 2016, 70, 905-919.

29 X. X. Zhang, Y. F. Fan, X. M. Tao and K. L. Yick, Crystallization and prevention of supercooling of microencapsulated $n$-alkanes, J. Colloid Interface Sci., 2005, 281(2), 299-306.

$30 \mathrm{~F}$. Cao and B. Yang, Supercooling suppression of microencapsulated phase change materials by optimizing shell composition and structure, Appl. Energy, 2014, 113, 1512-1518. 